\title{
Localization and identification of gas infrastructure defects by acoustic emission
}

\author{
Grzegorz Świt ${ }^{1, *}$, and Aleksandra Krampikowska ${ }^{1}$ \\ ${ }^{1}$ Kielce University of Technology, 25-314 Kielce, al. Tysiąclecia Państwa Polskiego 7, Polska
}

\begin{abstract}
Durability, reliability and usability are three foundations of reliable construction, crucial in economic and social context. Because localization and monitor of potential defect and assessment of its impact on condition of the construction are part of the durability assessment, we should develop appropriate methods, which would detect the beginning of technical condition process getting worse and could allow monitoring its progress during exploitation of the construction, not only at areas chosen subjectively or based on unverifiable computer models. One of this NDT methods complied with user expectations is acoustic emission method based on analysis of active destruction processes. The article presents results of $\mathrm{AE}$ applications to locate, monitor and identify destruction processes caused by exploitation burdens made to assessment the technical condition of gas infrastructure made from cast iron, steel and plastic.
\end{abstract}

\section{Introduction}

Diagnostic and monitoring construction condition are two important points in economic and social context. Aging infrastructure, deteriorating environmental conditions and rising exploitation burdens in engineering constructions are main stimulants for fast progressing researches on a new interdisciplinary field of knowledge known as "Structural Health Monitoring (SHM)", closely related to the safe structure use time [1].

In the assessment of durability of building structures two different problems are taken into account, one related to existing analysis of constructions designed in accordance with applicable standards, and the second associated with ensuring the anticipated period of use, considering load capacity and durability related features of newly designed structures $[2,3]$.

In this work, the authors' note focuses on the ability to assess the technical condition of the structure and its impact on the durability of existing gas infrastructure facilities (gas pipelines, bleed-stop units).

Pipeline transport system is widely used and recognized for the best way of transporting oil and gas in the energy industry, due to its safety and significant economic benefits. However, cases of pipeline failures have a very negative impact on people, environment and public safety. Normally, gas pipeline failures cause serious economic and image losses for gas infrastructure managers and sometimes lead to the death of outsiders. Due to the fact that gas pipelines are mainly operated underground, the infrastructure manager receives limited

*Autor do korespondencji: gswiter@gmail.com 
information on the actual technical condition of the gas network and data uncertainty makes it difficult to forecast and assess the risk of failure using one classic model based on method of Model Based Design (MBD). Therefore, in recent years, intelligent forecasting and risk assessment systems (IS) and in particular fuzzy inference systems (FIS) [4, 5] as well as artificial neural networks (ANNs) were elaborated and developed because of their possibility of inference based on uncertain and ambiguous data [6].

Important for the safety of the used infrastructure is to determine the beginning of the material damage process and structural elements, because attempts to stop once initiated processes are often ineffective, which leads to unforeseen disasters through unsealing or explosion of gas pipelines, which causes large material and social losses. Demand for a method capable of detecting the beginning of the destruction process and monitoring its progress in the entire capacity of the structure, not only in subjectively selected parts, complies with the acoustic emission technique. The method is based on a comparative analysis of acoustic emission signals registered during tests and collected in the database of reference signals corresponding to individual destructive processes. The results are used to identify and locate active destructive processes in gas infrastructure and in steel constructions, thus enabling global monitoring in the entire monitored area and recording only active developing lesions in real burden conditions [7-9].

This paper discusses examples of monitoring various types of gas pipelines (cast iron, steel, polyethylene) and transmission units using the acoustic emission method (AE).

\section{Acoustic emission in steel structures testing}

\subsection{Acoustic emission [1, 4-7]}

Every process in a material that leads to a decrease in internal energy generates AE signals (Fig. 1).

In the case of pipeline, the sources of acoustic waves are various types of damage, such as:

- corrosion processes of steel,

- crack initiation,

- fatigue crack evolution,

- steel yielding,

- friction between crack surfaces,

- leaks,

- extraneous noise.

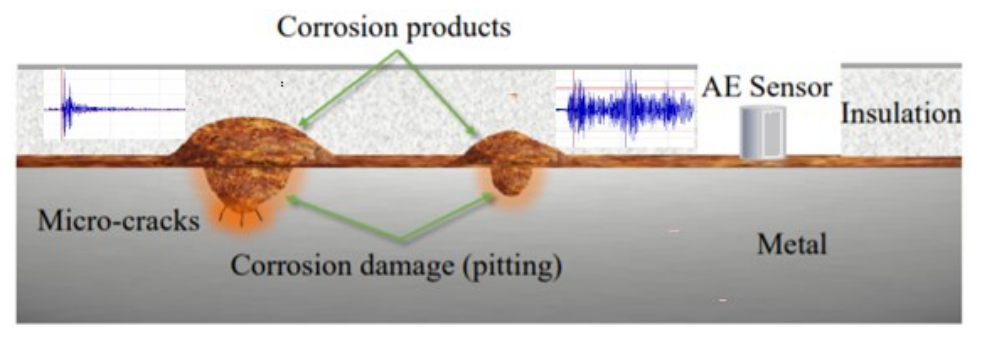

Fig. 1. Generating an AE signal by exemplary destructive processes on the gas pipeline element.

AE method allows for a comprehensive analysis of the object, which means, that damage is detected and located within a structure or a separate element, regardless of whether they are visible and regardless of their position in relation to the installed sensors. Simultaneously with revealing the damage, intensity of emerging emission signals indicates the level of danger that the defect is for the structure. 
The choice of acoustic emission as the research method was mainly determined by its advantages in relation to other non-destructive methods. These are:

- the possibility of locating these damages, which were not detected by traditional methods,

- the possibility of measurements in various working conditions, e.g. during exploitation burdens, trial burdens,

- the results of measurements and analysis can be obtained on an ongoing basis,

- $\mathrm{AE}$ is able to detect and identify different signal sources,

- $\mathrm{AE}$ is characterized by good intensity of damage development while using the object.

Analysing the individual stages of the measurement, according to Figure 1, waves generated by damage are recorded by acoustic emission sensors - converters that change the elastic wave to an electrical signal.

\subsection{Location of damage [7]}

An important element in $\mathrm{AE}$ measurements is the location of acoustic emission sources originating from destructive processes and other processes that accompany them.

In damage location, it is essential to apply the optimised spacing of sensors. Depending on the material, the propagation of AE waves decreases due to the structure of the material or damage and this process is known as attenuation. Attenuation can be calculated with an exponential relation described by the following equation (1):

$$
A_{f}=A_{O} \times e^{-\alpha d}
$$

where:

$A_{f}$ is the amplitude at the sensor position,

$A_{0}$ is the initial amplitude at the AE source,

$a$ is the damping factor, and

$d$ is the distance travelled by the wave [14].

Damping has a significant impact on the location of sources of destructive processes due to the fact that it is generally carried out based on the time difference of the signal coming from the localized source at the determined known wave propagation speed. In this way, you can locate the place where AE sources originate along a straight line (linear location). The method of locating the AE pulse source by measuring time of arrival has been successfully applied to locate faults on gas pipelines made of various types of materials. The TOA method consists in the placement of several sensors on the test element and the location of the wave source by comparing the time of arrival of signals to the sensors using the technique of trangulancy [15-18]. Location of the damage by registering the time of arrival is used when the signal will exceed the set threshold value. The amplitude threshold value is an important parameter in the analysis of AE signals, because it is characterising a point in which the system starts recording signals generated by destructive processes [19]. Often, the threshold value is used to eliminate noise and interference, but there is also danger that the signals generated by the damage will be ignored. In order not to make a mistake when establishing the threshold value, it is necessary to perform acoustic background measurement.

Figure 2 illustrates linear location used in damage quantification for pipeline joints.

If the distance between two sensors $\mathrm{S} 1$ and S2 is known and specified as D, the distance L1 between the source and the sensor S1 can be calculated determined from $(2-6)$ : 


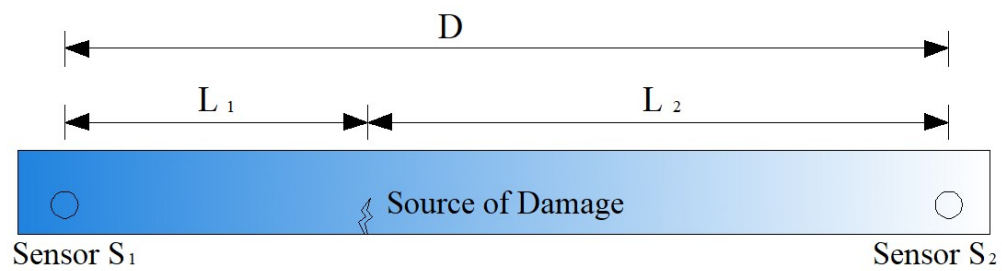

Fig. 2. Linear location scheme.

$$
\begin{gathered}
L_{1}=c \times T_{1} \\
L_{2}=c \times T_{2} \\
L_{1}-L_{2}=c\left(T_{1}-T_{2}\right)=-c \Delta t \\
L_{1}+L_{2}=D \\
L_{1}=0,5(D-\Delta t \times c)
\end{gathered}
$$

where: $T_{1}$ and $T_{2}$ these are the time when the signal reaches two sensors, $\Delta t$ is the difference between the times of coming $T_{1}$ and $T_{2}$, and $c$ is a velocity of AE.

The linear location makes it possible to determine the location of the signal source in a straight line between two sensors. The location is based on the interpolation of the signal arrival time to two sensors.

\subsection{AE signals database [6-7]}

Each destructive process is a source of acoustic emission which is characterized by the parameters of the recorded signal. The current traditional methods of AE signal analysis are burdened with significant errors resulting from the approximate character of the previously used theories and their implementation $[10,11]$. The theories and solutions used so far are burdened with many simplifying assumptions which consequently lead to significant errors in the evaluation of the propagating velocity of elastic wave forms especially for high frequency signals $[12,13]$. In addition, by using single parameters of acoustic emission and not having a formula, there is a great danger of making an incorrect decision regarding the technical condition of the tested structures and destructive processes causing them.

Therefore, to assess the technical condition using the acoustic emission method, it should be used on solutions based on statistical methods of signal clustering and formula recognition.

To build a base of reference signals in the method of identifying destructive processes in gas infrastructure (IDPGI) [6-7] the NOESIS 5.8 program is used, which uses hierarchical, non-hierarchical statistical clustering methods and neural networks.

To build a base of reference signals in the IDPGI method the FORGY algorithm was used as an example of analysis based on the fuzzy clustering method. It is helpful in case when it does not exist clear, sharp border separating natural groups of objects. In the real world, the phenomenon of multiple or partial belonging to a given object is often observed. Two types of fuzzy clustering can be distinguished: the first uses fuzzy relations to clustering; the second type is based on the criterion function, on the basis of which fuzzy groups are formed. A simplified scheme of the FORGY algorithm using fuzzy clustering consists in the following steps: 
1. determining the input parameters of the algorithm like the number of intervals $c$, the ending criterion $\boldsymbol{E}$, blur factor, membership coefficients $\boldsymbol{U}(\boldsymbol{0})$, cycle index $\boldsymbol{t}=\mathbf{1}$,

2. calculation of the vector formula $\boldsymbol{V}(\boldsymbol{t})$ and array $\boldsymbol{U}(\boldsymbol{t})$ according to formula (7) and (8)

$$
\begin{aligned}
& v_{i}=\frac{\sum_{i=1}^{N}\left(u_{i j}^{(t-1)}\right)^{m} x_{i}}{\sum_{i=1}^{N}\left(u_{i j}^{(t-1)}\right)^{m}} \\
& u_{i j}=\frac{1}{\sum_{j=1}^{c}\|\| \frac{x_{i}-v_{a}^{(t)}}{x_{i}-v_{j}^{(t)}} \| \frac{2}{m^{m-1}}}
\end{aligned}
$$

where: $m>1$ - dilution factor,

$c$ number of clusters,

$n$ - number of objects,

$v_{i} \in R$ formula $i-t h$ of this group,

$u_{i j}-$ affiliation $x_{j}$ to $i-t h$ of this group,

$t$ - iteration number,

3. if the new division matrix does not differ by more than $\varepsilon$ and the iteration limit has not been reached, please go back to step 2 .

The created base of reference IDPGI signals it allows to identify destructive processes on other constructions and then to use them to build a base of reference signals. It is possible to continuously extend and supplement the base of reference signals.

Table 1 shows the destructive processes with a colour and shape of the data point assigned to each AE signal to indicate the AE signal class.

Signal classes:

No 1 - rupture,

No 5 - perforation/deformation,

No 2 - friction,

No 6 - pitting,

No 3 - crack growth,

No 7 - corrosion,

No 4 - crack initiation,

No 8 - elastic behaviour.

Table 1. AE signal classes, symbols, risk codes and levels.

\begin{tabular}{|c|c|c|c|c|c|c|c|c|}
\hline Colour & & & & & & $\nabla$ & $\Delta$ & \\
\hline Class & No 1 & No 2 & No 3 & No 4 & No 5 & No 6 & No 7 & No 8 \\
\hline Risk code & 0 & 1 & 1 & 2 & 2 & 3 & 4 & 5 \\
\hline Risk level & very high & high & high & $\begin{array}{c}\text { higher } \\
\text { medium }\end{array}$ & $\begin{array}{c}\text { higher } \\
\text { medium }\end{array}$ & medium & low & no risk \\
\hline
\end{tabular}

The received standard file was used to assess the technical condition of sections of gas pipelines made of various materials. The exemplary results will be presented in the further part of the work.

\section{In-situ testing}

In studies of the technical condition of the gas pipeline sections and a blocking and bleeding system, a 24 channel acoustic emission processor $\mu$ Samos was used together with a set of preamplifiers and piezoelectric sensors with a frequency in the range of $30-80 \mathrm{kHz}$. Due to the measurement conditions (strong interference in the environment), the measuring threshold was set at $40 \mathrm{~dB}$. Before each measurement, the background measurement was performed and the correctness of the apparatus operation was checked using the Hsu-Nilsen formula. 
The tests were carried out on two sections of high and low pressure gas pipelines as well as a shut-off and bleed system during normal exploitation. The purpose of the research was the possibility to assess the technical condition of the gas infrastructure components under investigation and verification of the correctness of locating and identifying destructive processes by the INVESTOR. The measurement scheme is shown in Figure 2.

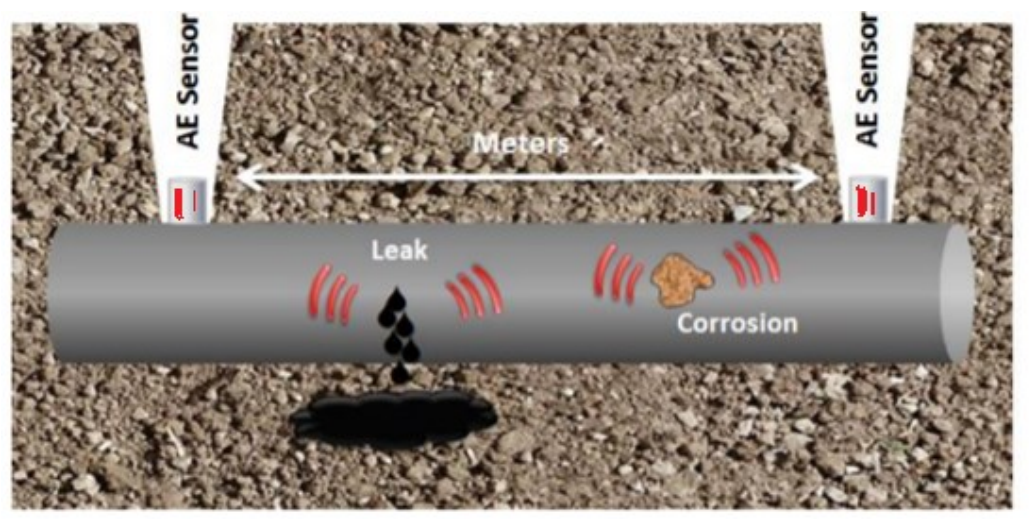

Fig. 3. Measurement scheme of a section of a gas pipe.

\subsection{Condition assessment by AE - High-pressure steel gas pipeline}

The tests were carried out on a 35-year old high-pressure gas pipeline built of steel pipes $300 \mathrm{~mm}$ in diameter welded together. The gas pipeline has passive anti-corrosion protection in the form of a bituminous coat and active protection in the form of cathodic protection. In a research a $200-\mathrm{m}$ fragment of a gas pipeline was tested in harsh environmental conditions such as: $80 \mathrm{~m}$ - strongly watered ground, $10 \mathrm{~m}$ - jumble under the road, $120 \mathrm{~m}$ - sand.

The location of the measurement is shown in Figure 4.

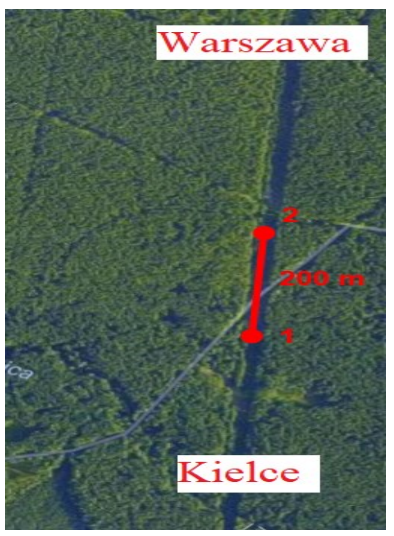

Fig. 4. Location of the measuring section of the steel gas pipeline.

The first measurement was made on the surface of pipes covered with bitumen protection and after removing it and cleaning the pipe surface. The purpose of this study was to evaluate the effect of bitumen on damping of the acoustic emission signal. It has been found out that it is possible to study sections of steel pipes in the ground up to $500 \mathrm{~m}$ long. Then the proper measurement was started. On the surface of the gas pipeline, two piezoelectric sensors with a flat characteristic in the frequency range of $30-80 \mathrm{kHz}$ were mounted in a distance of $200 \mathrm{~m}$. 
Based on the linear location, places with increased acoustic emission activity are indicated (Fig. 5).

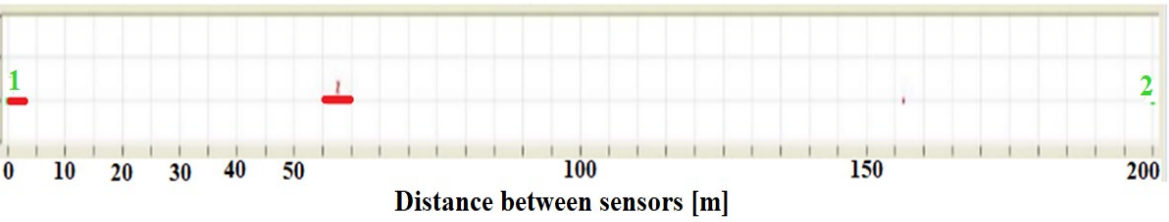

\section{Energy [eu]}

1301

Distance between sensors $[\mathrm{m}]$

100

$\mathbf{5 0}$

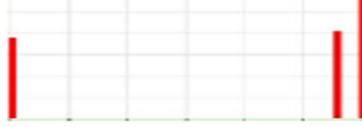

$\mathbf{0} \quad \mathbf{5 0}$

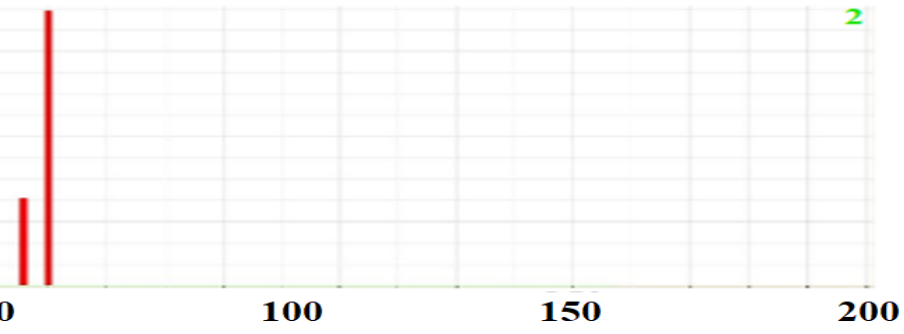

Distance between sensors $[\mathbf{m}]$

Fig. 4. Location of places with increased activity - energy histogram as a function of distance.

In order to identify destructive processes, registered signals were analysed using the IDPGI method's reference file. The results are shown in Figures 5 and 6.

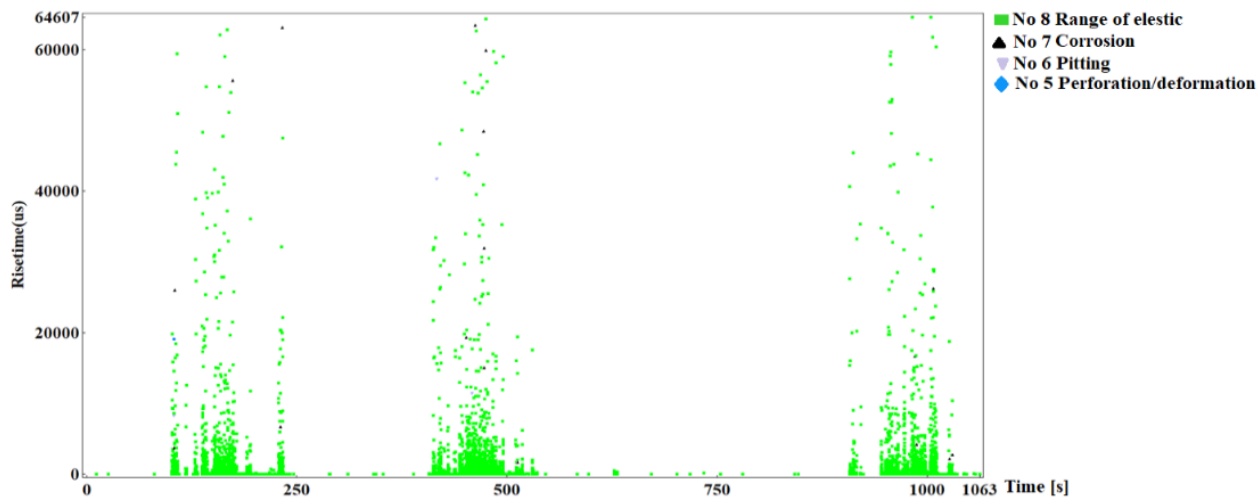

Fig. 5. Scatter plot of AE signal rise time as a function of time.

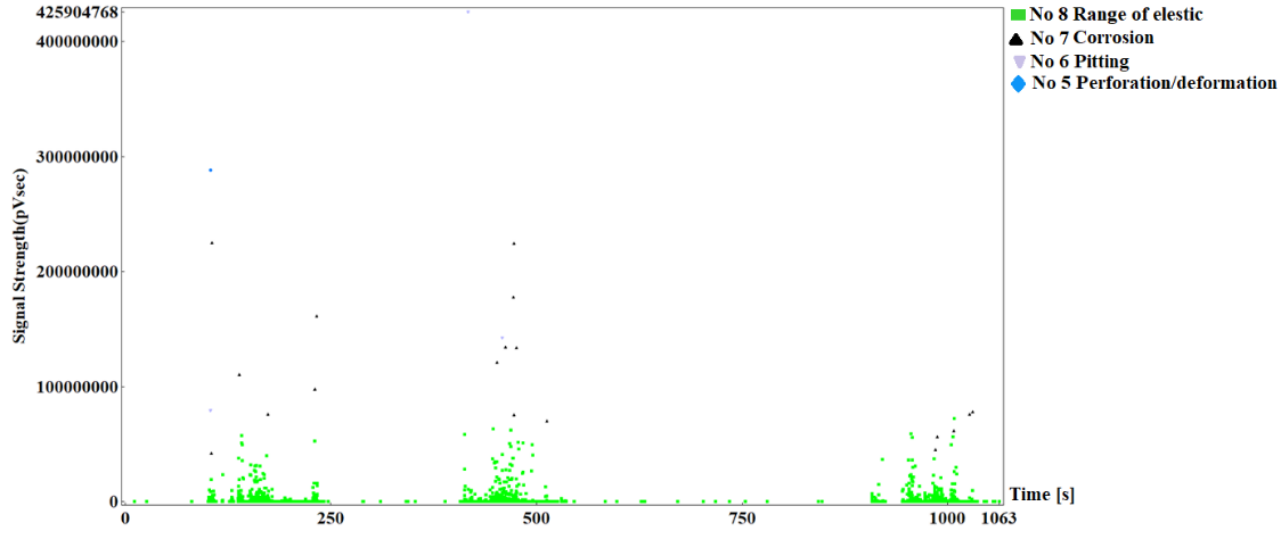

Fig. 6. Scatter plot of AE signal strength as a function of time. 
It should be noted that the analysis of the reference signal database allowed for the selection of four processes which generate AE signals. Signals marked with No. 8 and green colour indicate the work of the pipe in the scope of elastic operation and accompany the phenomena related to the operation of the gas pipeline. Appearing signals marked with No. 7, No. 6 and No. 5 indicate destructive processes related to corrosion centres in places indicated on the basis of location. Signals marked as No. 7 - black colour which rise time (Fig. 5) is in the range of $18,000 \mu \mathrm{s}$ to $65,000 \mu \mathrm{s}$ and their signal strength (Fig. 6) reaches values up to $1 \mathrm{E}+8 \mathrm{pVs}$ are associated with surface corrosion processes. However, if the rise time reaches $18000 \mu \mathrm{s}$, the signal strength is in the range from $1 \mathrm{E}+8$ to $2.2 \mathrm{E}+8 \mathrm{pVs}$ we are dealing with cracking processes of corrosion products and intergranular, pitting corrosion. It is worth noticing that for the acoustic emission signals generated by perforation processes, the rise time parameter reaches values up to $20,000 \mu$ s of signal strength ranging from $2.3 \mathrm{E}+8$ to $3.2 \mathrm{E}+8$. This phenomenon means changes in the structure of the material. Recorded signals are generated not continuously but initiated by the operational load. The resulting signals suggest that the strain caused by the exploitation burden is in a safe range. The only place exposed to the uncontrolled increase of destructive processes is a place at a distance of 55 to $60 \mathrm{~m}$ under the forest road, after which intensive traffic of vehicles with a mass above 40T takes place. The location indicated an area of increased intensity and 3 destructive processes. The remaining part of the gas pipeline can be safely used.

\subsection{Condition assessment by AE - Low-pressure gas pipeline made of PE 80}

The tests were carried out on a 10-year low-pressure gas pipeline constructed of polyethylene PE 80 pipes, $60 \mathrm{~mm}$ diameter, joined together by welding. The gas pipeline has active protection in the form of cathodic protection. The test was carried out on a length of $\mathrm{L}=750 \mathrm{~m}$ of a gas pipeline operating under normal environmental conditions: the entire gas pipeline laid at a depth of about $120 \mathrm{~cm}$ in sandy soil. The location of the measurement is shown in Figure 7.

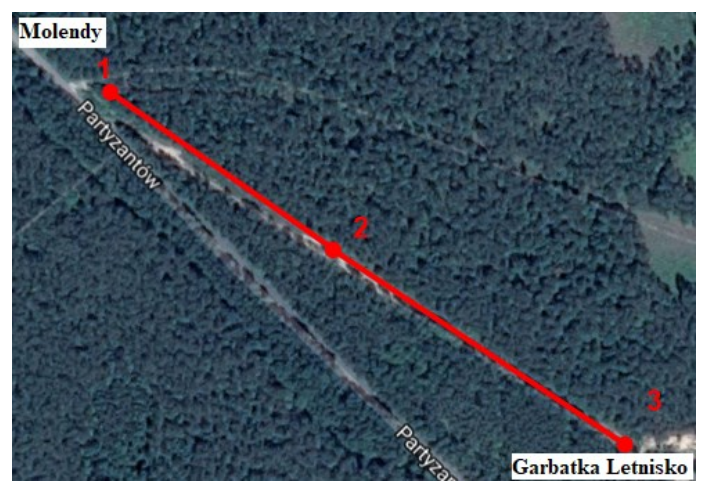

Fig. 7. Location of the measuring section of the polyethylene gas pipeline (PE 80).

The first measurement was made on the surface of the gas pipeline to assess the impact of the material (PE 80) on the damping of the acoustic emission signal. It was found out that it is possible to study sections of polyethylene pipes in the ground up to $500 \mathrm{~m}$ long. Then the proper measurement was started. On the surface of the gas pipeline, 3 sensors with flat characteristics were installed in the frequency range of $30-80 \mathrm{kHz}$ with a spacing of 300 and $450 \mathrm{~m}$.

Based on the linear location, places with increased acoustic emission activity have been indicated (Fig. 8). 


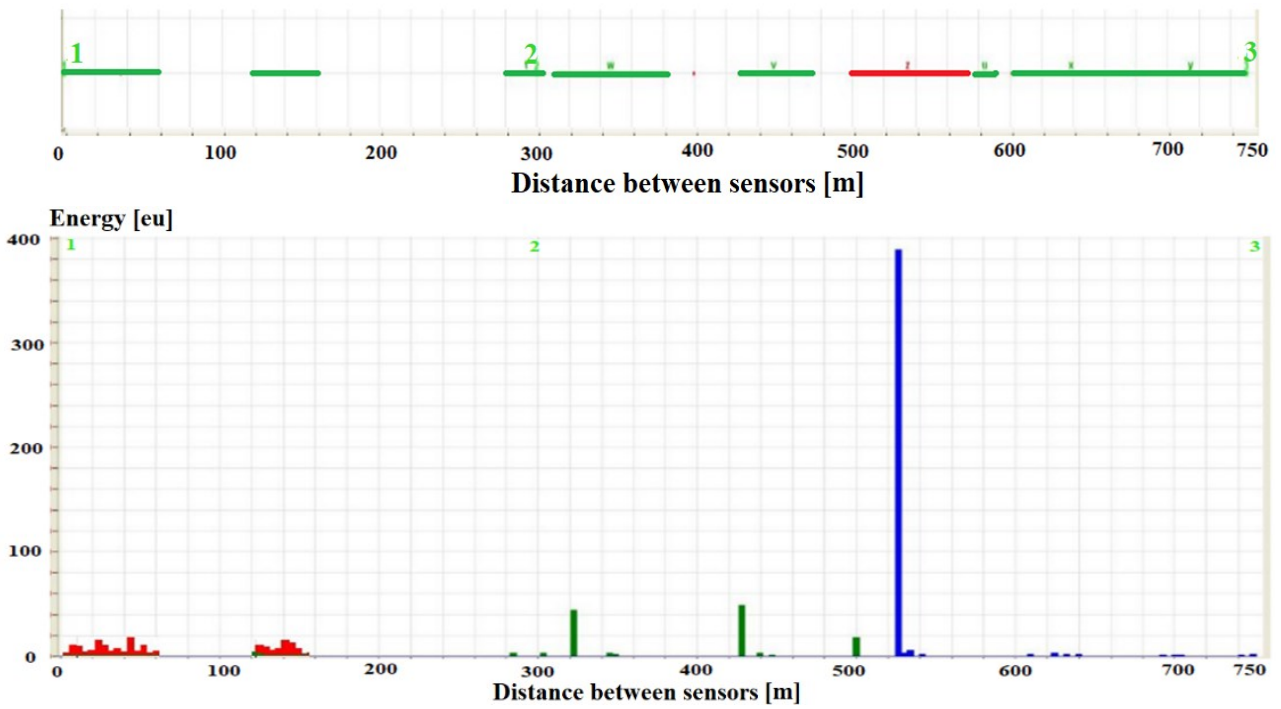

Fig. 8. Location of places with increased activity - energy histogram as a function of distance.

In order to identify destructive processes, registered signals were analyzed using the IDPGI method reference file. The results are shown in Figures 9 and 10.

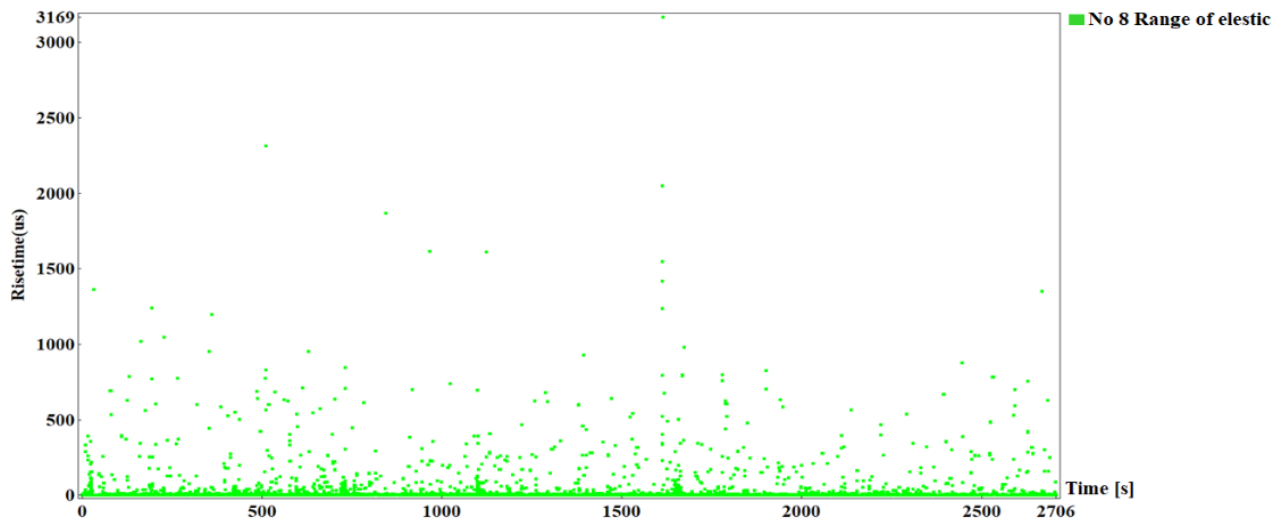

Fig. 9. Scatter plot of AE signal rise time as a function of time.

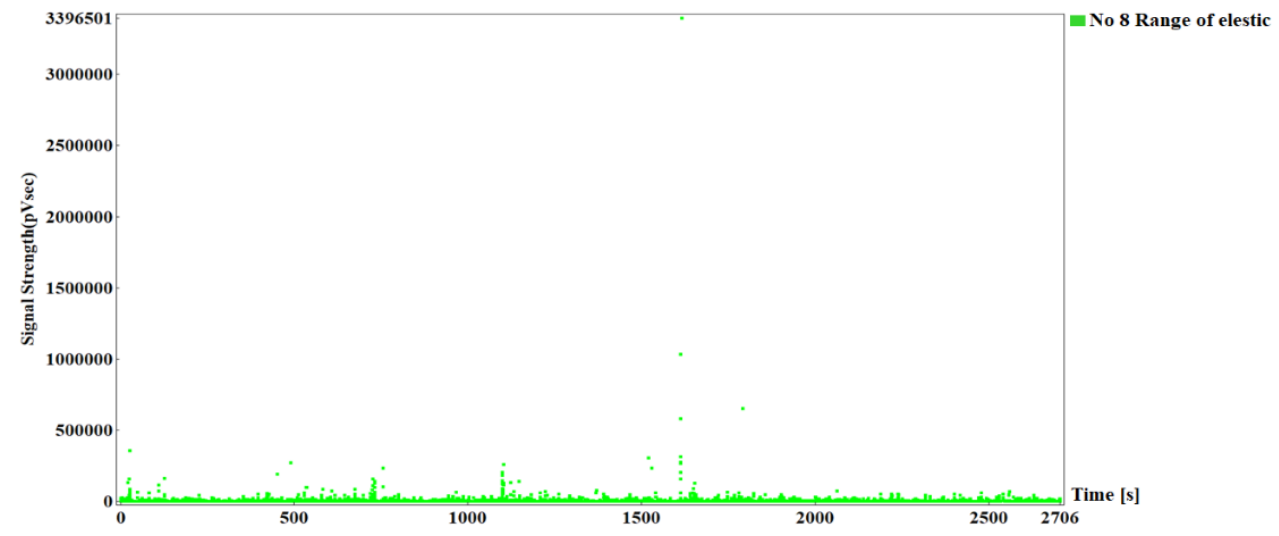

Fig. 10. Scatter plot of AE signal strength as a function of time. 
It should be noticed that the analysis of the base of reference signals allowed to select only one type of signal marked with No. 8 and green color which means the work of the pipe in terms of elastic operation and accompany phenomena related to the operation of the gas pipeline. These signals are characterized by a low rise time parameter (Fig. 9) up to 3,000 $\mu \mathrm{s}$ and a very low signal strength (Fig. 10) reaching sporadically $3.4 \mathrm{E}+6 \mathrm{pVs}$. Recorded signals are generated continuously and are accompanied by an exploitation burden caused by the gas and the gas flow. The resulting signals suggest that the stress caused by the exploitation burden is in a safe range.

\subsection{Condition assessment by $\mathrm{AE}-\mathrm{n}$-line shut off and relief valve system}

The tests were carried out on a ca. 35-year n-line shut off and relief valve system (Fig. 11) of two threads of a high-pressure gas pipeline built of $300 \mathrm{~mm}$ diameter steel pipes. The gas pipeline has passive anti-corrosion protection in the form of paint coatings and active protection in the form of cathodic protection. The research involved a $5 \mathrm{~m}$ system consisting of shut-off valves, ball valves, breathers, gauges and insulating connectors presented in Figure 12.

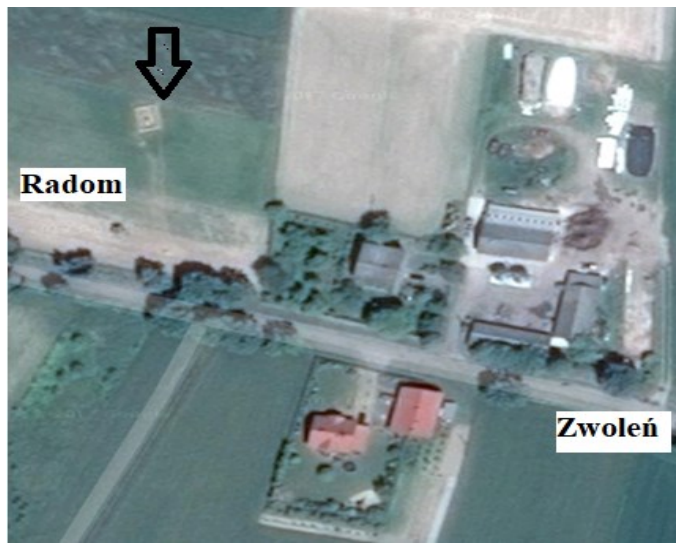

Fig. 11. Location of the $n$-line shut off and relief valve system.

On the surface of the components of the pipeline-bleed system of the gas pipeline, $5 \mathrm{~m}$ spacing, two piezoelectric sensors with flat characteristics in the $30-80 \mathrm{kHz}$ frequency range were installed.

Based on the linear location, places with increased acoustic emission activity have been indicated (Fig.12).

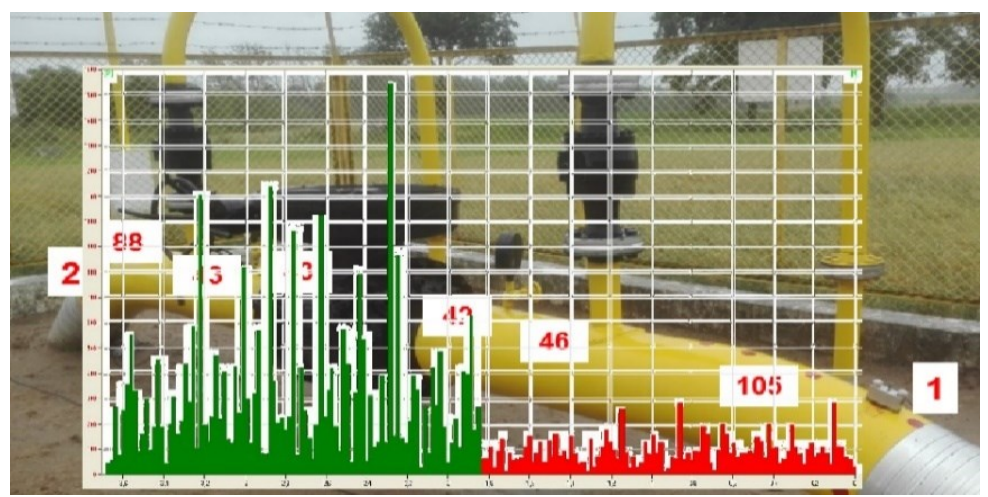

Fig. 12. Location of places with increased activity - energy histogram as a function of distance. 
In order to identify destructive processes, registered signals were analyzed using the IDPGI method reference file. The results are shown in Figures 13 and 14.

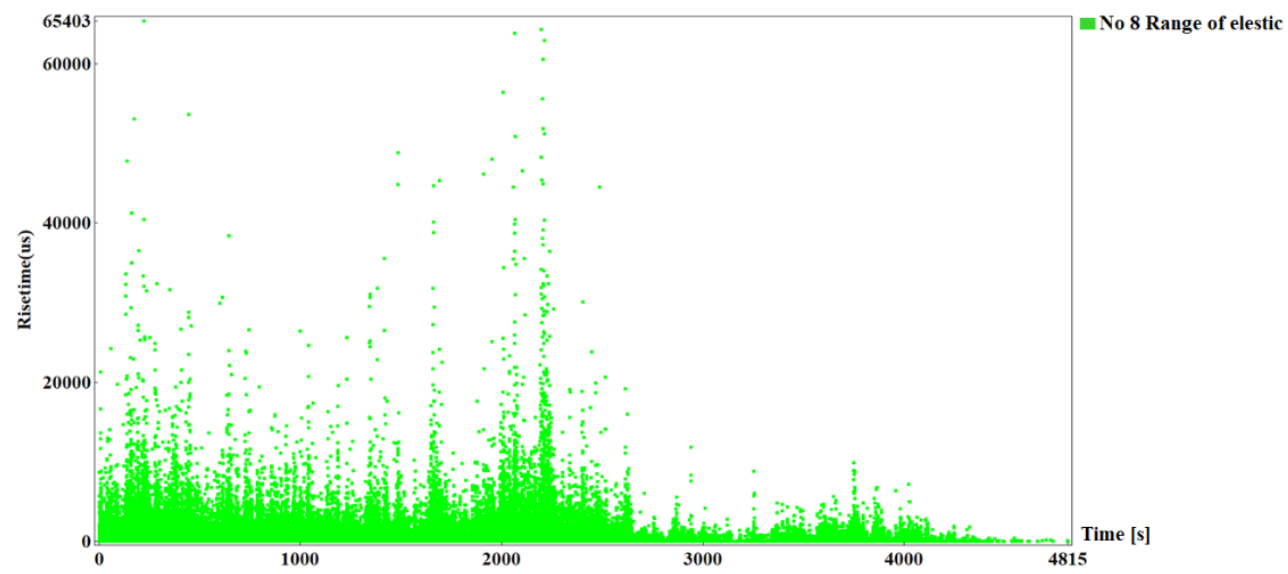

Fig. 13. Scatter plot of AE signal rise time as a function of time.

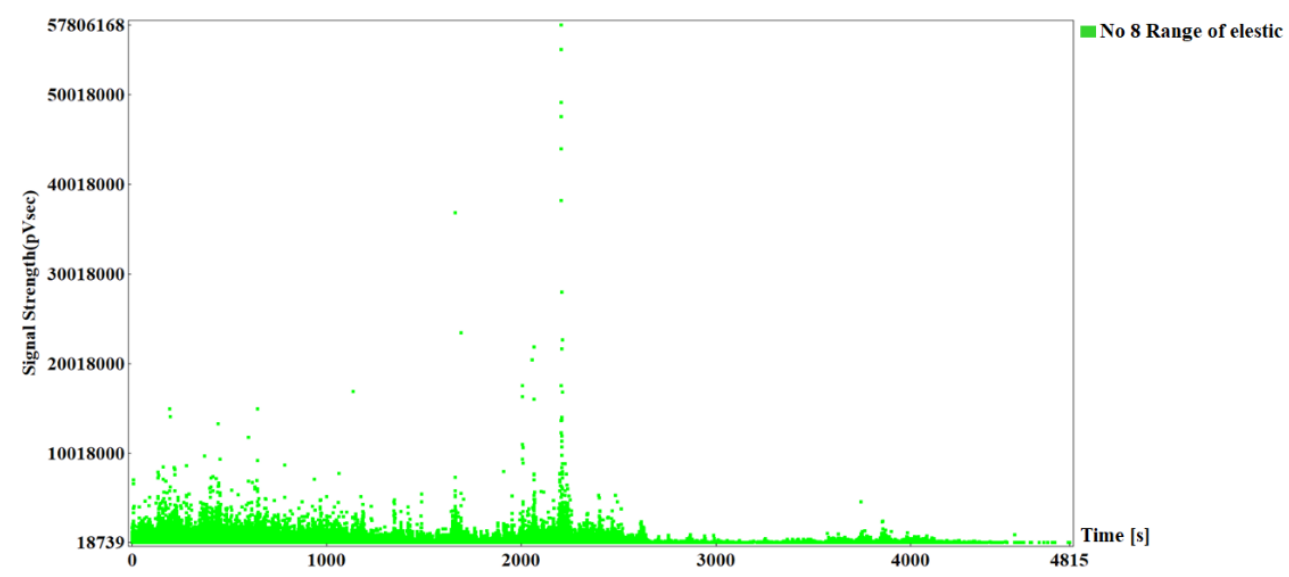

Fig. 14. Scatter plot of $A E$ signal strength as a function of time.

Observing Figure 12, it can be seen that there is an area on the left side of the gate that generates a large number of acoustic emission signals. Therefore, the registered data file was subjected to the analysis of the base of reference signals, which allowed to select only one type of signal marked with No. 8 and green color, which indicate the work of the pipe in the scope of elastic work and accompany phenomena related to the operation of the gas pipeline. These signals are characterized by a low rise time parameter (Fig. 13) up to 3,000 $\mu$ s and a very low signal strength (Fig.14) reaching sporadically 3.4E $+6 \mathrm{pVs}$. However, signals with higher parameters are related to mechanical failures of ball valves (valve leaks) and do not affect work safety, but generate large material losses for users of this shut-off and relief valve system. The recorded signals are generated continuously and are accompanied by an exploitation burden caused by the gas flowing and the poor work of the components of this system. 


\section{Conclusion}

1. The PE pipeline does not show signs of destructive processes threatening its safe operation - very good condition,

2. The pipeline from steel pipes outside the indicated places is in a good technical condition enabling safe work, however in a few places a corrosive process started on the surface of the gas pipeline (section about $60 \mathrm{~m}$ ) working in the deeply watered ground,

3. In the indicated place (pic.4) with increased AE activity, which is located on the border of the road and drainage ditch, after the discovery there was a damage to passive corrosion protection, a place of surface corrosion and pitting corrosion, which confirmed the results obtained during the measurements,

4. The pipeline survey shows the full usefulness of the IDPGI method for monitoring and assessment of their technical condition,

5. Optimal measuring sections for steel pipelines are $200-300 \mathrm{~m}(\max 500 \mathrm{~m})$ for polyethylene pipelines is $300-500 \mathrm{~m}(\max 700 \mathrm{~m})$,

6. Research on shut off and relief valve system shows the full usefulness of the method for assessing the tightness of these network elements.

\section{Ministry of Science}

and Higher Education

Republic of Poland

The project is supported by the program of the Minister of Science and

Higher Education under the name: "Regional Initiative of Excellence" in 2019 - 2022 project numer 025/RID/2018/19 financing amount|PLN 12,000,000. 


\section{References}

1. K. Ono, Diagnostics, 2(56) (2011)

2. Guideline : Guide for Service Life Design of Buildings. Part1 - General Principles. ISO Draft No 2, (International Standard Organization, 1995)

3. Service life prediction - state of the art report. ACI365.1R-00 (American Concrete Institute, Farmington Hills, USA, 2000)

4. G. Świt, A. Adamczak, A. Krampikowska, IOP Conf. Series: Mater. Sci. Eng., 245, 022031 (2017)

5. G.Świt, A.Adamczak, A.Krampikowska, IOP Conf. Series: Mat. Sc. and Eng., 251, 012002 (2017)

6. G. Świt, A. Krampikowska, Proceedings of the PHM-Chengdu Conference (2016)

7. G. Świt, Appl. Sci., 8(1295), (2018)

8. A. Deraemaeker, E. Reynders, G. De Roeck, J. Kullaa, Mechanical Systems and Signal Processing, 22(1), pp. 34-56 (2008)

9. F.N. Catbas, Structural Health Monitoring: Applications and Data Analysis Structural Health Monitoring of Civil Infrastructure Systems (Woodhead Publishing, 2009)

10. H.A. Elfergani, R. Pullin, K.M. Holford, Constr. Build. Mater. 40, pp. 923-933 (2013)

11. C. Xiwang, Y. Yong, M. Yifan, M. Lin, H. Xiaojuan, Sensors and Actuators, A 237, pp. 107-118 (2016)

12. D. Shantanu, S. Shibayan, J. Loss Prev. Process. Ind 41, pp. 17-106 (2016)

13. L.Yu, S.Z.Li, Appl. Acoust., 120, pp. 85-89 (2017)

14. H. Vallen, NDTnet, 7(9), (2002)

15. G. Fu, Inspection and monitoring techniques for bridges and civil structures (Woodhead Publishing Limited and CRC, Sawstone, UK, 2005)

16. P. Nivesrangsan, J.A. Steel, R.L. Reuben, Mech. Sys and Signal Proc., 21(2) (2007)

17. D. Gagar, P. Foote, P. Irving, Smart Mater. Struct., 23(10), pp. 105033 (2014)

18. J.J. Scholey, P.D. Wilcox, M.R. Wisnom, M.I. Friswell, M. Pavier, M.R. Aliha, J. Acoust. Emiss., 27, pp. 291-298 (2009)

19. J.J. Scholey, P.D. Wilcox, M.R. Wisnom, M.I. Friswell,. Ultrasonics, 49(6-7), pp. 538-548, (2009). 